\title{
Expression analysis of flavonoid biosynthesis genes during Arabidopsis thaliana silique and seed development with a primary focus on the proanthocyanidin biosynthetic pathway
}

\author{
Christiane Katja Kleindt ${ }^{1,2}$, Ralf Stracke ${ }^{1}$, Frank Mehrtens ${ }^{1}$, Bernd Weisshaar ${ }^{1 *}$
}

\begin{abstract}
Background: The coordinated activity of different flavonoid biosynthesis genes in Arabidopsis thaliana results in tissue-specific accumulation of flavonols, anthocyanins and proanthocyanidins (PAs). These compounds possess diverse functions in plants including light-attenuation and oxidative stress protection. Flavonoids accumulate in a stimulus- and/or development-dependent manner in specific parts of the plant. PAs accumulate in the seed coat (testa).

Findings: We describe the biological material and the preparation of total RNA for the AtGenExpress developmental silique and seed series. AtGenExpress ATH1 GeneChip expression data from the different stages were reanalyzed and verified using quantitative real time PCR (qPCR). We observed organ-specific transcript accumulation of specific flavonoid biosynthetic genes consistent with previously published data and our PA compound accumulation data. In addition, we investigated the regulation of PA accumulation in developing $A$. thaliana seeds by correlating gene expression patterns of specific flavonoid biosynthesis genes with different seed embryonic developmental stages and organs and present two useful marker genes for isolated valve and replum organs, as well as one seed-specific marker.

Conclusions: Potential caveats of array-based expression data are discussed based on comparisons with qPCR data. Results from ATH1 microarray and qPCR experiments revealed a shift in gene activity from general flavonoid biosynthesis at early stages of seed development to PA synthesis at late (mature) stages of embryogenesis. The examined PA accumulation-associated genes, including biosynthetic and regulatory genes, were found to be exclusively expressed in immature seeds. Accumulation of PAs initiates at the early heart stage of silique and seed development. Our findings provide new insights for further studies targeting the PA pathway in seeds.
\end{abstract}

\section{Background}

Flavonoids are aromatic amino acid-derived secondary metabolites with a multitude of roles in plants. For example, they act as antimicrobial agents, light protection pigments, feeding deterrents against pathogens and herbivores [1] and are, among other pigments, crucial for flower coloration, attracting insects for pollination and seed dispersal [2]. Flavonoids are subdivided into several classes, with three major ones in Arabidopsis

\footnotetext{
* Correspondence: bernd.weisshaar@uni-bielefeld.de

'Bielefeld University, Department of Biology, Genome Research, 33594 Bielefeld, Germany

Full list of author information is available at the end of the article
}

thaliana: flavonols, anthocyanins, and proanthocyanidins (PAs, condensed tannins) [reviewed in [3]]. Flavonols contribute to UV-protection $[4,5]$, while the red and purple anthocyanin pigments play a role in lightattenuation and serve as antioxidants [reviewed in [6]]. PAs are also effective antioxidants [7], and, in A. thaliana, responsible for the brown color of seed coats (testa) [reviewed in [3]] subsequent to their oxidation $[8,9]$.

Mature seeds consist of the embryo, the end product of embryogenesis, surrounded by the seed coat [reviewed in [10]]. During embryogenesis, the fertilized egg cell (zygote) divides and differentiates into the 
following main stages: four cell stage, globular stage, heart stage, torpedo stage, walking-stick stage, curled cotyledon stage, and green cotyledon stage [[11], reviewed in [12]]. Recent studies have shown that silique and seed development are highly coordinated processes which involve embryogenesis and endosperm development, as well as the maternal development of the seed coat and siliques hulls $[13,14]$. The testa of A thaliana seeds mainly consist of the inner and outer integument. PAs exclusively accumulate in the innermost inner integument (the endothelium cell layer), the micropylar area and the chalazal bulb of $A$. thaliana seeds $[9,15,16]$. Mutants defective in PA accumulation, referred to as transparent testa $(t t[17,18])$, have yellow to pale brown colored seeds [15].

Figure 1 presents a simplified, schematic representation of the flavonoid biosynthesis pathway in A. thaliana with a main focus on PA biosynthesis. These metabolites are synthesized in a series of enzymatic steps beginning with chalcone synthase (CHS), which synthesizes naringenin chalcone from 4-coumaroyl-CoA and malonyl-CoAs. Activities of the enzymes chalcone isomerase (CHI), flavanone 3-hydroxylase (F3H), and flavonoid 3'hydroxylase $\left(\mathrm{F}^{\prime} \mathrm{H}\right)$ result in the synthesis of dihydroflavonols. The enzyme dihydroflavonol 4-reductase (DFR) reduces dihydroflavonols to leucoanthocyanidins which are in turn converted to anthocyanidins by the activity of leucoanthocyanidin dioxygenase (LDOX). Anthocyanidins form a branching point in the flavonoid biosynthesis synthesizing anthocyanins (via glycosylation by UDP-dependent glucosyltransferases (UGTs)) or epicatechin (by the enzyme anthocyanidin reductase (ANR; BANYULS, BAN [19])). Epicatechin is the precursor of PAs [19], formed via the involvement of different enzymes including glutathione $\mathrm{S}$ transferase (GST), a multidrug and toxic compound extrusion-type transporter (MATE), a P-type $\mathrm{H}^{+}$-ATPase (AHA10), and a laccase-like (LAC). Genes encoding these proteins are collectively referred to as PA biosynthetic genes, although, for instance, the transporter is relevant for localization and not synthesis. Steps in late PA synthesis have yet to be elucidated [20].

The expression of flavonoid biosynthesis genes is regulated by a hierarchy of different transcription factors (TFs), as reviewed in Lepiniec et al. [3]. The accumulation of PAs and anthocyanin compounds in A. thaliana is mainly regulated at the level of transcription by a heterotrimeric complex, composed of the WD40 protein TRANSPARENT TESTA GLABRA1 (TTG1), a basic helix-loop-helix (bHLH), and a R2R3-MYB (MYB) TF, which interacts with target promoters of flavonoid biosynthesis genes [reviewed in [21]]. In A. thaliana seeds, PA biosynthesis is known to be under the control of TTG1, TT8 (bHLH42), and TT2 (MYB123) [22-24]. In addition, the zinc-finger protein TT1 has been shown to be involved in seed-specific PA accumulation [25]. Anthocyanin biosynthesis is mainly regulated by TTG1, which interacts with several bHLHs (GLABRA3 (GL3), ENHANCER OF GLABRA3 (EGL3), TT8) and the R2R3-MYBs (PRODUCTION OF ANTHOCYANIN PIGMENT1 (PAP1, MYB75), PAP2 (MYB90), MYB113, and MYB114) [26].

Flavonoid accumulation has been widely used to study gene regulation in plants due to tissue-specific regulation of flavonoid biosynthesis genes in response to a variety of stress conditions [reviewed in [27]], as well as during development [reviewed in [28]]. Here, we present expression profiles of flavonoid biosynthesis genes during silique and seed development in A. thaliana. We relate expression data of flavonoid-, and PA biosynthesis genes in particular, with specific seed developmental stages. Finally, our organ-specific transcript data (from silique hulls and isolated seeds) provides useful markers for tissue-specific gene expression studies.

\section{Results and Discussion}

Correlation of silique length to embryo developmental stages and timing of proanthocyanidin accumulation in the testa during seed development

Silique development was followed using light microscopy, focusing on stages of embryo development (according to Bowman [11]) in enclosed immature seeds. Figure 2 shows the correlation of the different stages of embryogenesis (listed in Table 1) to silique length at defined growth conditions, as well as to PA accumulation during seed development of wild type $A$. thaliana Col-0. The accumulation of PAs in the seed coat endothelium was visualized using vanillin staining.

PA accumulation was first detected at developmental stage 3 , corresponding to mid globular to early heart stage (Figure 2) and continued until the embryo turned green (stages 9-10). The observed timing of PA accumulation during seed development in A. thaliana Col-0 (Figure 2) is consistent with that noted by Debeaujon $e t$ al. [16] in the Wassilewskija accession.

\section{Verification of silique and seed developmental series ATH1 GeneChip data by quantitative real time PCR}

As part of the AtGenExpress expression atlas, RNA from eight developmental stages (stages 3-10 with siliques containing seeds of the stages 3-5 and isolated seeds of the stages 6-10; see Table 1 and Methods) were used for ATH1 GeneChip experiments [29]. We reproduced the biological samples used for the AtGenExpress developmental series (see Methods) for quantitative real time PCR (qPCR) analyses. The siliques containing seeds and isolated seed samples provide a limited resolution of silique and seed developmental gene 


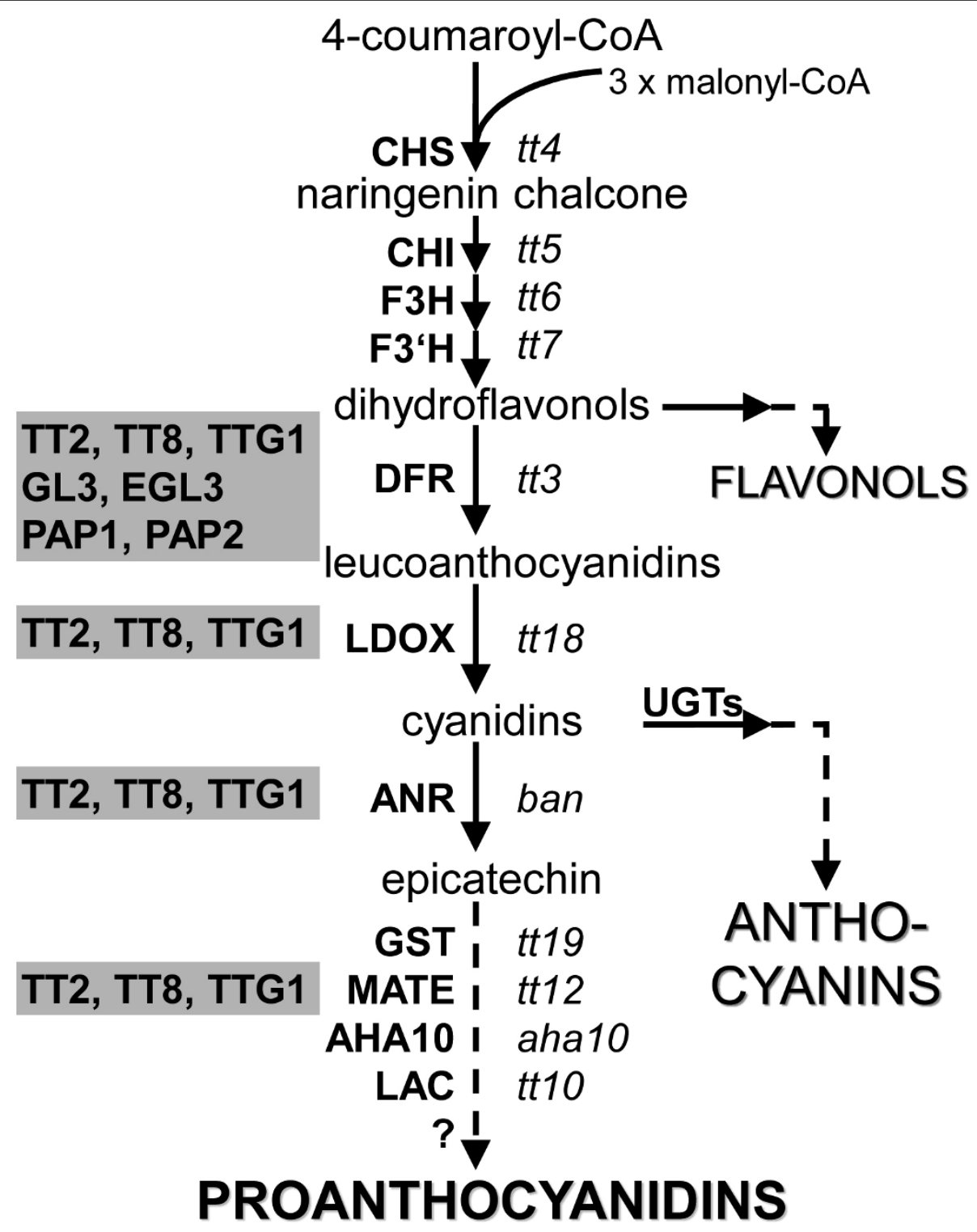

Figure 1 Simplified, schematic representation of flavonoid biosynthesis in A. thaliana with a focus on proanthocyanidins. Essential steps of the flavonoid biosyntheses leading to proanthocyanidins (PAs) in the seed are presented. Enzymes and transcriptional regulators (grey shaded) are indicated by bold, capital letters; corresponding mutant plant lines are in italics. Dashed arrows tag multiple steps which are not fully understood. The question mark indicates a limited knowledge of this biosynthetic step and a restricted understanding of the involvement of the associated factors. Abbreviations are as follows: ANR/BAN anthocyanidin reductase, AHA1O P-type $\mathrm{H}^{+}$-ATPase, CHS chalcone synthase, CHI chalcone isomerase, DFR dihydroflavonol 4-reductase, EGL3 enhancer of glabra3, F3 H flavanone 33-hydroxylase, F3'H flavonoid 3'-hydroxylase, GL3 glabra3, GST glutathione S transferase, LAC laccase-like, LDOX leucoanthocyanidin dioxygenase, MATE multidrug and toxic compound extrusion-type transporter, PAP production of anthocyanin pigments, $\pi$ transparent testa, $\Pi$ G transparent testa glabra, UGTS UDP dependent glycosyltransferases. Modified from [1,67,68].

expression. Therefore, to increase resolution and determine organ specificity of gene expression, we included samples from earlier stages of silique development (0-2; Table 1), and from different parts of developing siliques (stages 6-10; Figure 2), such as isolated seeds and isolated silique hulls consisting of valves and replum organs.
We checked for reproducibility and validity of the developmental series data using qPCR, which is generally accepted as the standard $[30,31]$ and more sensitive method [32,33] for verification of microarray gene expression data. In general, $\mathrm{qPCR}$ results verified the array results. However, in some cases, differences in expression were observed between the two techniques, 


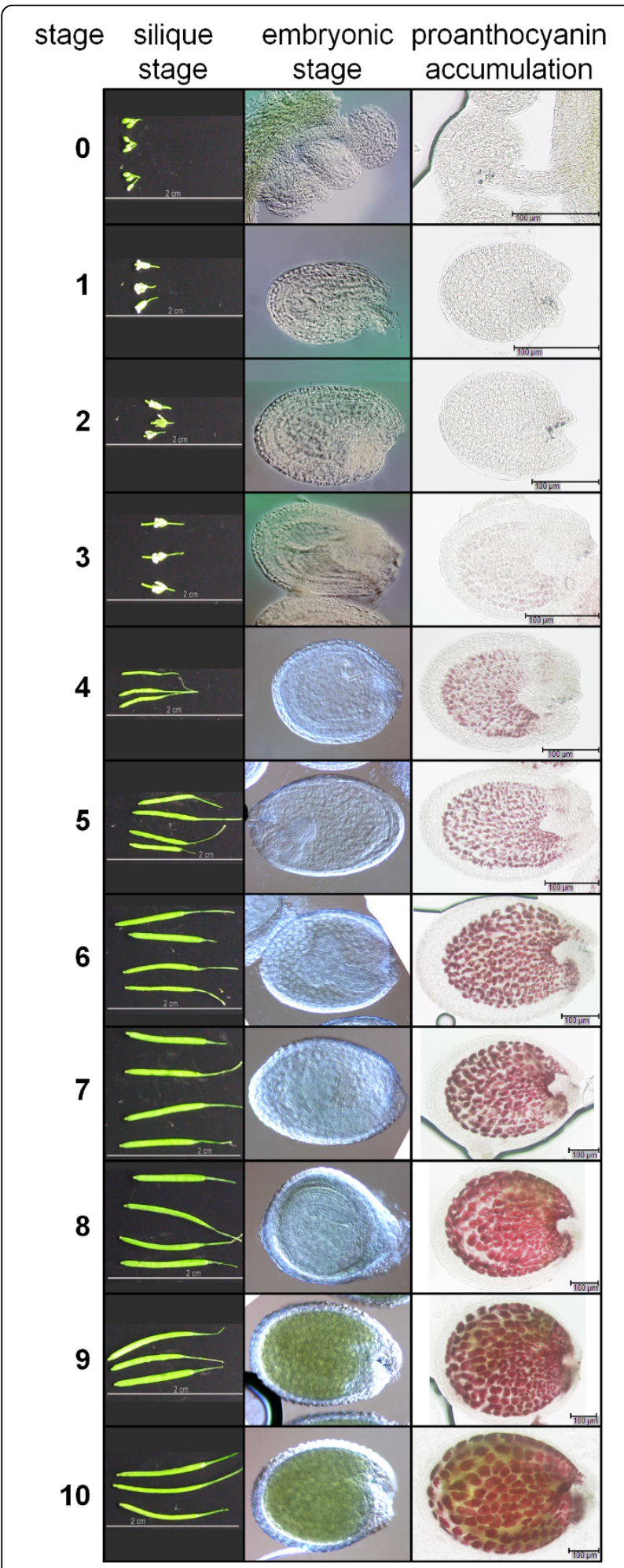

Figure 2 Developmental stages of $A$. thaliana siliques and single seeds, and accumulation of proanthocyanidins. Developmental stages of $A$. thaliana siliques are correlated to embryonic stages of single seeds according to Bowman [11], and to the accumulation of proanthocyanidins in the seed coat using vanillin staining. reflecting the limitations of using arrays for gene expression studies. These discrepancies may be due to: i) variations in biological samples, ii) hybridization-based sequence specific effects [34], and/or iii) signal saturation during ATH1 hybridization. For example, expression of the CHS gene is controlled by several different stimuli [reviewed in [3]] acting on the biological samples. Therefore, the ATH1 GeneChip expression profile of $C H S$ is probably difficult to verify by qPCR.

In addition, cross hybridization can occur within multiple genes resulting in nonspecific gene expression profiles [35]. We found that the ATH1 gene expression profile of $L D O X$ represented transcripts of two related A. thaliana genes (leucoanthocyanidin dioxygenase, putative (AT4G22870); LDOX (AT4G22880)). However, qPCR expression data specifically represented only the LDOX gene. Finally, signal saturation during ATH1 hybridization may also prevent accurate verification of gene expression by qPCR [36,37]. This was observed for ARABIDOPSIS THALIANA SEED GENE3 (ATS3) and TT10. Although qPCR results for these two genes did not match the array results, they were consistent with previously published data.

A correlation analysis of genes showing a non saturated, gene-specific ATH1 expression profile reveals a good linear correlation $(r=0.52)$ of ATH1 GeneChip expression data with qPCR transcript levels $\left(\log _{2}\right.$ transformed) using the Pearson product-moment correlation coefficient (PMCC, $r$ ).

\section{Evaluation of specificity of isolated seed, valve and replum organs by organ-specific expression analysis}

To evaluate organ specificity of the isolated samples, we selected two genes with known valve and replum-specific expression (ALCATRAZ, ALC and RESPONSIVE TO DEHYDRATION20, RD20) and one with a seed-specific expression pattern (ATS3) [38]. ALC is expressed in valve margins of the silique, as shown by GUS promoter studies [39] and RD20 transcripts are present in siliques but not in seeds, as shown by Northern blot analysis [40]. In our study, expression of $A L C$ and RD20 genes was typically higher in isolated valve and replum organs compared to silique containing seeds and very low in isolated seed samples (Figure 3A). Low transcript levels were detected in isolated seed sample leading back to sample separation method. In contrast, a late seed-specific expression was observed for ATS3 (Figure 3A).

Although separation of small siliques (stages 6-8; Figure 2) into seeds, valves and replum is difficult with immature seeds sticking to the replum, we were able to detect organ-specific gene expression patterns using qPCR and confirmed that our separation procedure was successful since minimal variations in corrected crossing points (cCPs) were observed for $A L C, R D 20$, and $A T S 3$ 
Table 1 Definition of stages for A. thaliana seed and silique development used in this work.

\begin{tabular}{|c|c|c|c|c|c|}
\hline stage & stages of embryogenesis & $\begin{array}{l}\text { hours after } \\
\text { flowering }\end{array}$ & $\begin{array}{c}\text { stage length }^{1} \\
{[\mathrm{~mm}]}\end{array}$ & $\begin{array}{l}\text { sample harvested for ATH1 GeneChip } \\
\text { hybridization }\end{array}$ & $\begin{array}{l}\text { AtGen-Express accession } \\
\text { (ATGE) No. } \\
\end{array}$ \\
\hline 0 & mature embryosac & 0 & $1.2-1.4$ & - & - \\
\hline 1 & up to four cells & $12-24$ & $2.0-2.2$ & - & - \\
\hline 2 & early globular to mid globular & $24-48$ & $2.4-2.8$ & - & - \\
\hline 3 & mid globular to early heart & $48-66$ & $3.8-4.2$ & siliques containing seeds & ATGE_76 \\
\hline 4 & early heart to late heart & $66-84$ & $6.0-6.8$ & siliques containing seeds & ATGE_77 \\
\hline 5 & late heart to mid torpedo & $84-90$ & $8.0-9.2$ & siliques containing seeds & ATGE_78 \\
\hline 6 & mid torpedo to late torpedo & $90-96$ & $10.0-11.2$ & isolated seeds & ATGE_79 \\
\hline 7 & $\begin{array}{l}\text { late torpedo to early walking- } \\
\text { stick }\end{array}$ & $96-108$ & $11.6-12.2$ & isolated seeds & ATGE_81 \\
\hline 8 & $\begin{array}{l}\text { walking-stick to early curled } \\
\text { cotyledons }\end{array}$ & $108-120$ & 12.4- 13.0 & isolated seeds & ATGE_82 \\
\hline 9 & $\begin{array}{l}\text { curled cotyledons to early } \\
\text { green cotyledons }\end{array}$ & $120-144$ & $12.8-13.6$ & isolated seeds & ATGE_83 \\
\hline 10 & green cotyledons & 144-192 & $13.6-14.4$ & isolated seeds & ATGE_84 \\
\hline
\end{tabular}

${ }^{1}$ Minimal and maximal flower and silique length observed under our green house conditions.

(Additional file 1). This was not previously resolved in the ATH1 GeneChip data set. Moreover, these three genes serve as useful markers for isolated seed (ATS3), and valve/replum organs $(A L C, R D 20)$.

\section{Reanalysis of the ATH1 silique and seed developmental series reveals stage-specific expression patterns of flavonoid biosynthesis genes}

We evaluated embryo developmental stage-specific expression profiles using the previously generated ATH1 GeneChip dataset [29]. A heat map analysis of tissue specific marker genes, principle component analysis of the seed developmental samples, as well as developmental analyses of the ATH1 GeneChips, which show two main, opposing expression trends from early to late seed stages, have been presented and discussed in Schmid et al. [29].

First, differentially expressed genes were obtained $\left(\log _{2}\right.$ foldchange $\geq 1.0$ or $\log _{2}$ foldchange $\leq-1.0, p \leq 0.05$ ) for each developmental stage by comparing expression at each stage to that at stage 10 (Figure 3B). Second, MapMan [41] was used to identify enriched functional processes at each stage. The highest number of differentially expressed genes was detected at mid globular to early heart stage (stage 3) with 8021 genes exhibiting significantly altered expression at this stage. These results were highly similar to the AtGenExpress expression atlas data [29].

We also found that expression patterns of flavonoid biosynthesis genes were dramatically altered, with a majority of these genes down-regulated during silique and seed development in the ATH1 GeneChip datasets (data not shown).

In addition, gene expression data was consistent with the onset of PA accumulation in the seed coat at the early heart stage, detected using vanillin staining (Figure 2). Similar results were reported in an ATH1 expression profile study conducted on developmental stages 1-3 in the Landsberg erecta accession [13] showing a continuous activation of the PA branch. Our analysis also shows that PA accumulation does not occur at early developmental stages (0-2, Table 1$)$.

Using qPCR, we also examined the gene expression profile of the flavonoid biosynthesis regulator TT1 that is not represented on the ATH1 chip. We found that TT1 transcripts are exclusively present in developing young seeds (data not shown), similar to that reported by Sagasser et al. [25].

\section{Expression of flavonoid biosynthesis genes is seed- specific}

We also examined the organ-specific expression patterns of flavonoid biosynthesis genes during silique and seed development using qPCR. The flavonoid biosynthetic genes CHS, DFR, LDOX, and BAN (Figure 4A-D) were expressed in isolated seeds consistent with the ATH1 GeneChip isolated seeds expression data. Likewise, the PA biosynthesis-specific genes TT19, TT12, AHA10, and TT10 (Figure 4E-H) exhibit isolated seed-specific expression [16]. Seed-specific expression of TT10 has been previously reported using semi-quantitative reverse transcription PCR (RT-PCR) by Pourcel et al. [42]. Although ATH1 and qPCR results do not account for expression differences within different parts of the seed, it is conceivable that the detected seed-specific expression of PA biosynthetic genes is due to expression in the testa where PAs are primarily known to accumulate [16]. Only basal transcript levels of these genes were detected in isolated valves and replum organs suggesting 

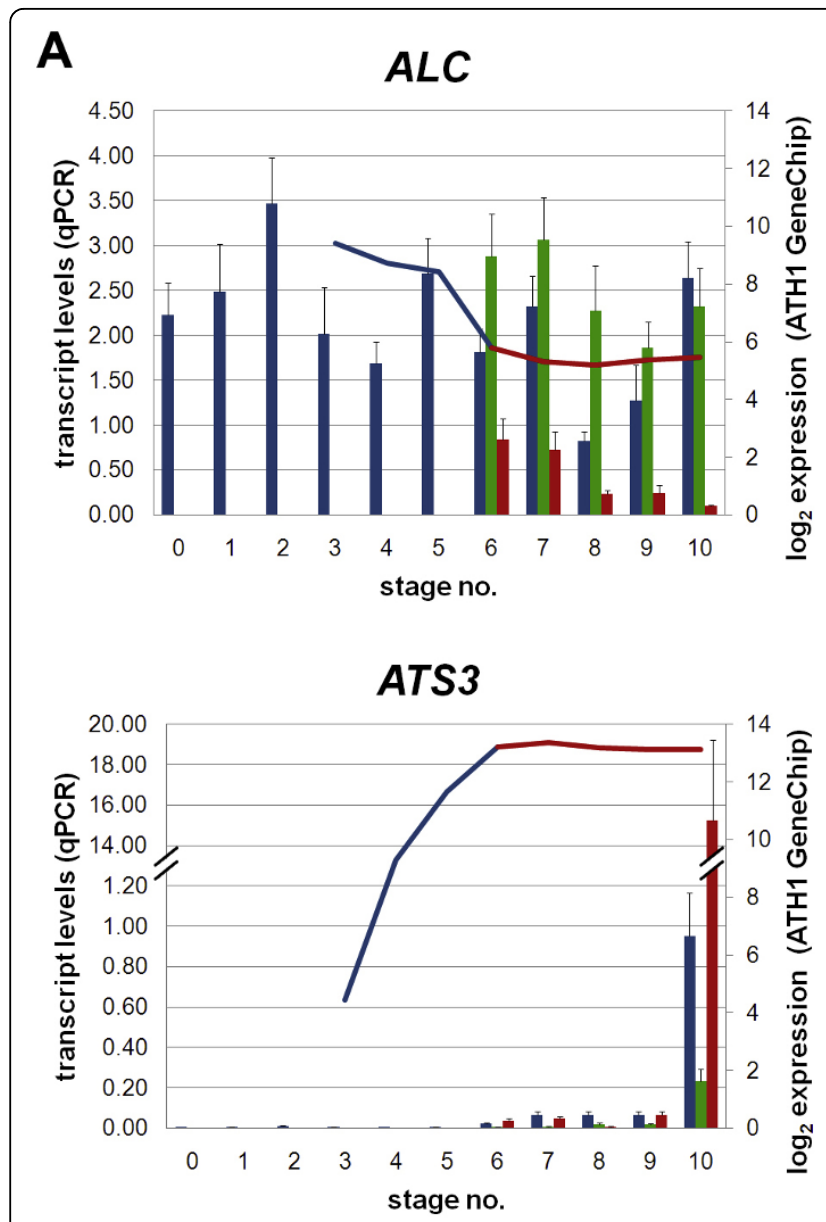

B

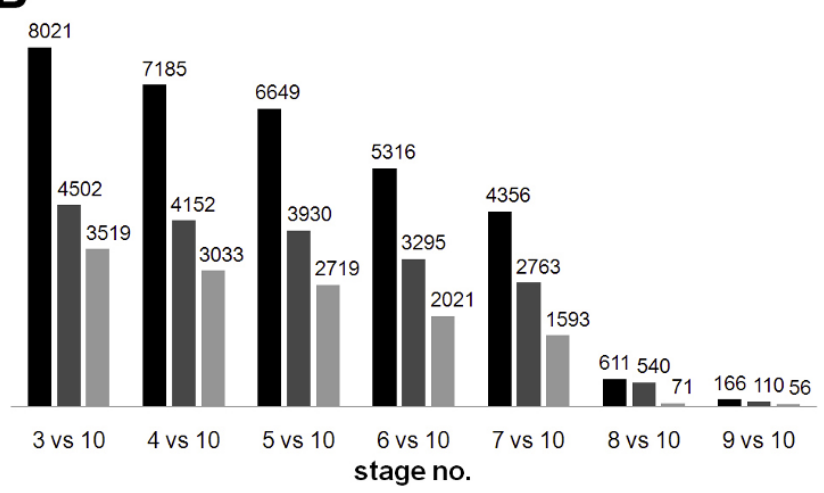

RD20

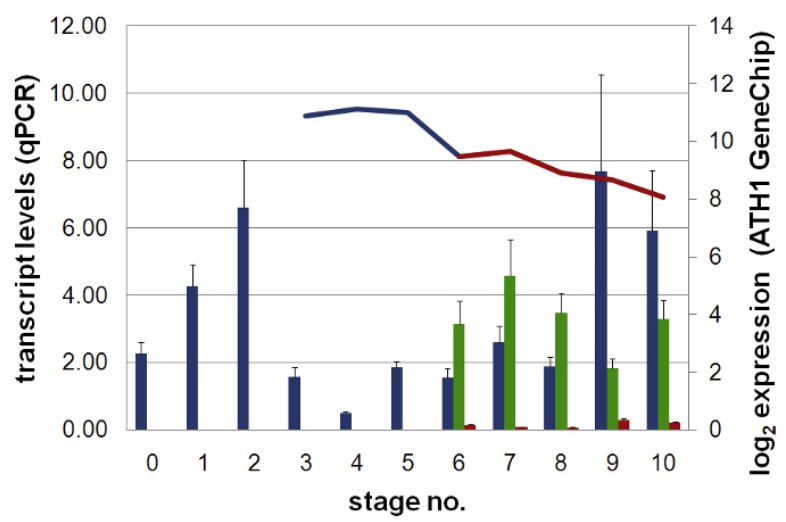

- siliques containing seeds - isolated valves and replum - isolated seeds

Figure 3 Organ-specific expression of selected marker genes. (A) qPCR transcript levels are presented as bars (relative expression levels shown on the primary axis were normalized to TIM44-2), while corresponding $\log _{2}$ expression values of ATH1 GeneChips are displayed as curves on the secondary axis. ALC is expressed in the valves margins of the silique [39]. RD20 is expressed specifically in siliques, but not in seeds [40]. ATS3 expression is seed-specific [38]. The presented genes are useful markers for separated A. thaliana silique samples (isolated valve and replum organs, isolated seeds). (B) ATH1 GeneChip hybridization data were used to determine the number of differentially regulated genes at each developmental stage normalized to stage 10. The number of significantly up- and down-regulated genes is shown ( $\log _{2}$ foldchange $\geq 1.0$ or $\log _{2}$ foldchange $-1.0, p \leq 0.05)$. Color key: blue, siliques containing seeds; green, isolated valves and replum organs; red, isolated seeds. Abbreviations are as follows: ALC alcatraz, RD20 responsive to dehydration 20, ATS3 A. thaliana seed gene3, TIM44-2 translocase inner membrane subunit 44-2. 


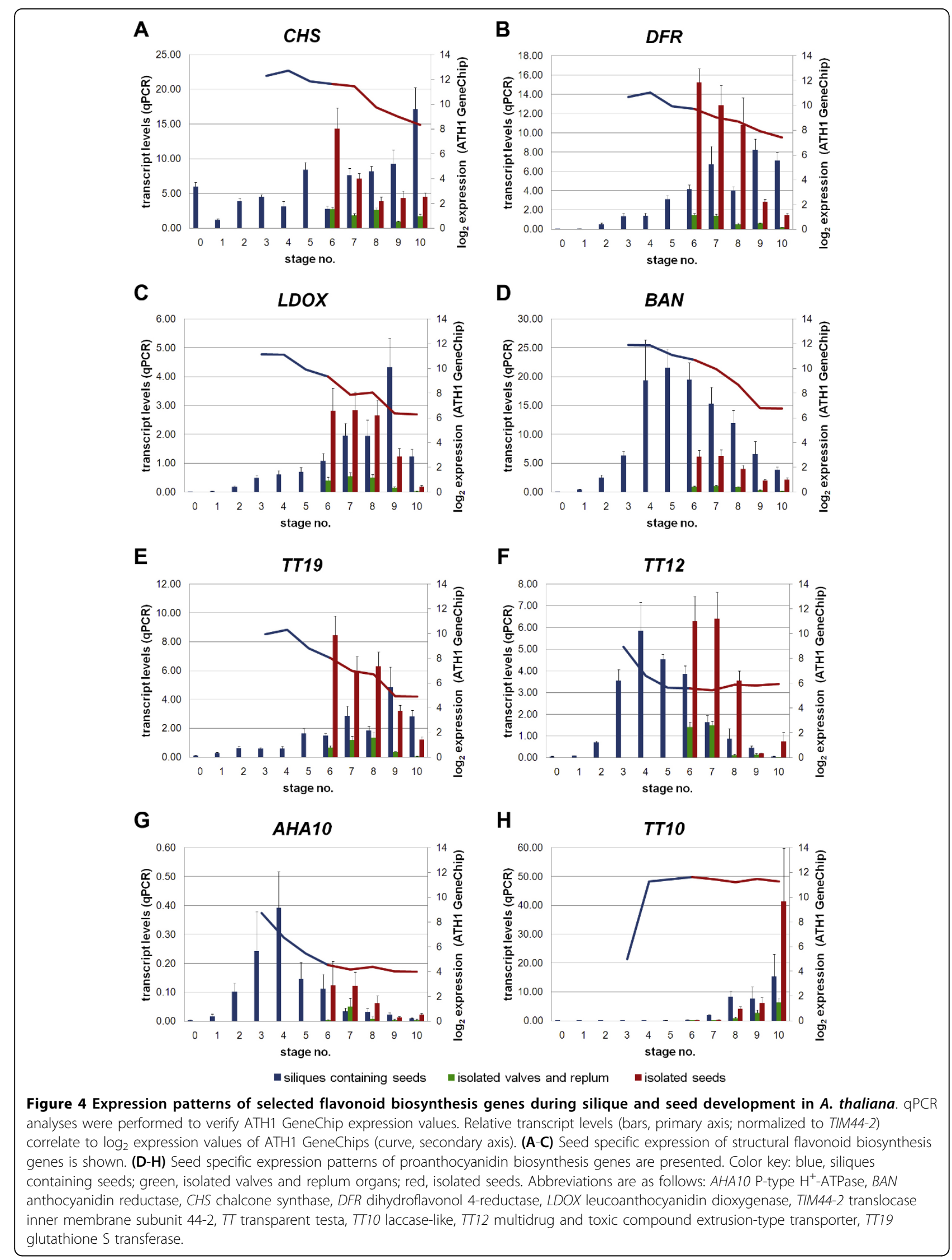


that PA accumulation-associated genes are not expressed in silique hulls.

\section{TT2 and TT8 show organ-specific gene expression in isolated seeds}

During A. thaliana seed development, PAs accumulate specifically in the endothelium layer of the seed coat $[8,43]$ via the activity of at least four regulatory TFs: TT2, TT8, TTG1, and TT16 [23,44]. These TFs are required for the expression of several flavonoid biosynthesis genes in maturing seeds [22,23], such as DFR and $B A N$ (Figure 1, 4B, D).

We studied gene expression profiles of TT2, TT8, TTG1, and TT16 during silique and seed development (Figure 5A). TT2 is specifically expressed during early and mid developmental stages in siliques containing seeds (stages 4-7; Table 1), as previously reported by Nesi et al. [23]. Highest TT2 expression was observed in isolated seeds reflecting an activation of flavonoid biosynthesis towards PA accumulation. Expression of TT8 also appears to be isolated seed-specific, whereas TTG1 exhibits low level gene expression in all organs. The seed-specific expression of these genes reflects well the known regulation of PA biosynthesis by the heterotrimeric TTG1-TT8-TT2 complex [reviewed in [3]].

TT8 and TTG1 are also involved in the regulation of anthocyanin accumulation in vegetative tissues $[22,45]$. Since anthocyanin levels in the harvested plant material were below our detection limit, we concluded that the anthocyanin pathway was not activated (data not shown). This is supported by our qPCR results, since only very low transcript levels were detected for both genes in isolated valve and replum organs.

TT16 is expressed in immature siliques (stages 1 and 2 ), consistent with previously published RT-PCR results [44] and ATH1 expression profile results in the Landsberg erecta accession [13], and in isolated seeds. However, very low expression was detected in valves and replum organs.

\section{Genes involved in anthocyanin accumulation are} activated during early stages of seed development

Although we could not measure detectable amounts of anthocyanins in the plant material harvested for qPCR experiments, we also looked at the expression profiles of anthocyanin biosynthesis genes that showed faint transcript levels in silique hulls (valves and replum organs). Figure $5 \mathrm{~B}$ shows that gene expression levels of $P A P 1$ and $U G T 75 C 1$ are similar to that observed byATH1 GeneChips.

qPCR at earlier time points revealed a specific increase in PAP1 gene expression at developmental stages 1 and 2 (Figure 5B), consistent with ATH1 expression profile studies in the Landsberg erecta accession [13]. Likewise,
UGT75C1 showed increased gene expression at early stages (Figure $5 \mathrm{~B}$ ), indicating a potential regulation by PAP1. This is in agreement with Tohge et al. [46] who reported an induction of $U G T 75 C 1$ gene expression in PAP1 over-expressing plants.

In summary, we describe the biological material and the preparation of RNA samples for the AtGenExpress developmental silique and seed series. We successfully verified selected ATH1 GeneChip expression data using quantitative real time PCR (qPCR) on a biological replicate. We also included earlier developmental stages and addressed organ specificity (silique hulls, isolated seeds) by qPCR, both of which were not analyzable in the ATH1 GeneChip data. We present two useful marker genes for isolated valve and replum organs, as well as one seed-specific marker showing significant organ specific differences in expression.

We reanalyzed the ATH1 GeneChip silique and seed developmental series and found that expression levels of flavonoid biosynthesis genes are dramatically altered during development. Similar to the ATH1 GeneChip data, our qPCR results showed that PA biosynthesis-associated gene expression increases specifically in maturing seeds. Our data not only supports previous results on PA accumulation, but also shows that accumulation of PAs initiates at the early heart stage of silique and seed development providing a key finding for future studies targeted at investigating the PA pathway in seeds.

\section{Methods}

\section{Plant growth conditions}

For ATH1 GeneChip experiments, Arabidopsis thaliana seeds (Columbia-0 accession; Col-0) were sown on a peat-based substrate (70\% ground white peat; $20 \%$ vermiculite $3-6 \mathrm{~mm} ; 10 \%$ washed sand; $100 \mathrm{~g} / \mathrm{m}^{3}$ iron chelate; $100 \mathrm{~g} / \mathrm{m}^{3}$ Radigen $^{\odot}$ trace elements), stratified in the dark for 3 days at $4^{\circ} \mathrm{C}$ and then transferred to the greenhouse. Plants were grown under short day conditions (8 $\mathrm{h}$ light/16 h dark cycles) for 3 weeks and then long day conditions (16 h light/8 h dark cycles) with additional illumination using SIL-lamps (Na- and $\mathrm{Hg}$-steam, 180$200 \mu \mathrm{E}$ ) with $50-60 \%$ humidity and mean temperature of $18^{\circ} \mathrm{C}$. Plants were harvested 8 weeks after germination.

For qPCR experiments, A. thaliana Col-0 plants were grown as described above with minor modifications. Plants were grown on peat-based substrate $(70 \%$ white peat $0-8 \mathrm{~mm}, 20 \%$ vermiculite $3-6 \mathrm{~mm}, 10 \%$ sand, $1.4 \mathrm{~g} /$

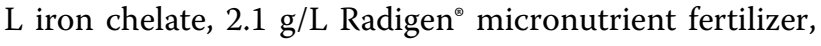
$\mathrm{pH} 5.5-6.0)$ with a mean temperature of $20^{\circ} \mathrm{C}$.

\section{Harvest and stage confirmation of plant material for Affymetrix GeneChip experiments}

To ensure harvest of homogenous samples, seed developmental stages were confirmed by light microscopy 


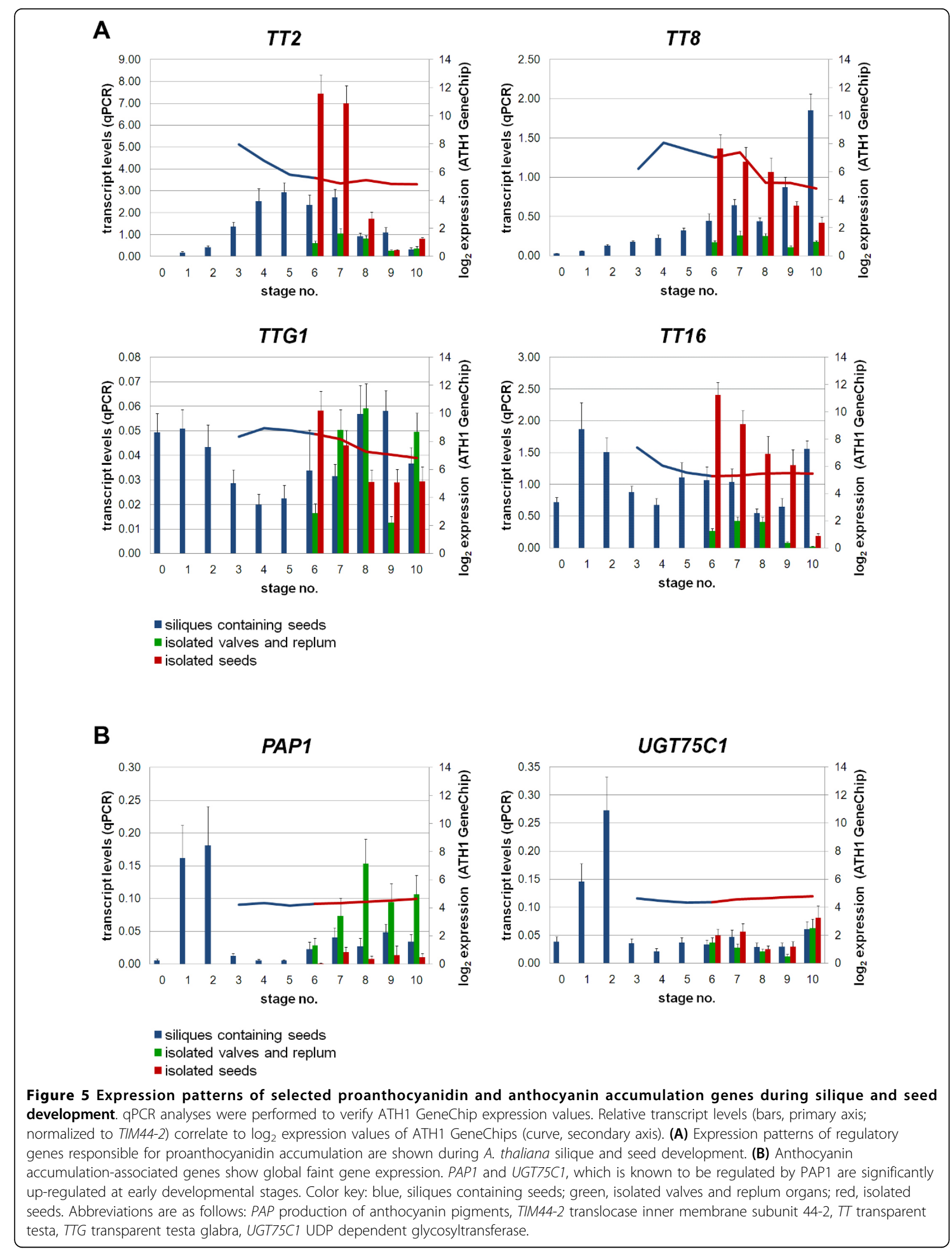


according to Bowman [11], as shown in Figure 2. Seeds originating from at least four different siliques per developmental stage were cleared prior to microscopy in a mixture of chloral hydrate/glycerol/water $(8: 2: 1)$ for at least $2 \mathrm{~h}$ at room temperature. Microscopy was performed using a Nikon Eclipse E600 microscope system (Nikon GmbH, Düsseldorf, Germany). Batches of up to $100 \mathrm{mg}$ of developing siliques (developmental stages 3-5 (see Table 1) or immature seeds isolated from opened siliques (developmental stages 6-10) were harvested on dry ice, transferred into liquid nitrogen and stored at $-80^{\circ} \mathrm{C}$ until RNA isolation. For assignment to the various stages we focused on stages of embryo development (Figure 2) to ensure harvest of homogenous developmental stages. Anatomic terms for the description of plant organs were determined according to the Plant Ontology ${ }^{\mathrm{rm}}$ Consortium [47].

\section{RNA extraction for Affymetrix GeneChip experiments}

Extraction of total RNA was performed according to the protocol of Ruuska and Ohlrogge [48] with the following modifications: the extraction buffer contained water instead of tri-isopropylnaphtalene sulphonic acid, and the 2-butoxyethanol/isopropanol precipitation was performed only once. RNA resulting from this extraction procedure was purified using RNeasy Mini spin columns (Qiagen, Hilden, Germany) according to the manufacturer's protocol.

\section{Affymetrix GeneChip hybridization, data pre-processing and expression analyses}

Total RNA extracted from eight developmental stages (siliques containing seeds, stages 3-5, and isolated seeds, stages 6-10; see Table 1) were used for Arabidopsis ATH1 GeneChip (Affymetrix UK Ltd., High Wycombe, UK) hybridizations. The labeling, hybridization, and array scanning were performed by Detlef Weigel's group (MPI of Developmental Biology, Tuebingen, Germany) according to Affymetrix's GeneChip Expression Analysis Technical Manual [49]. Microarrays were processed in triplicate for each developmental stage as part of the AtGenExpress expression atlas [29]. Table 1 presents the main stages of embryogenesis, the harvested sample organs, and the AtGenExpress accession number of the microarray datasets produced with the RNA samples described above. MIAME information for all samples, as well as microarray raw data are publicly available from AtGenExpress [29] at TAIR [50].

Microarray raw data of three biological replicates per developmental stage were analyzed using EMMA 2 [51] and the Bioconductor 2.4 software package [52]. Default settings were used for all Bioconductor modules. Expression values $\left(\log _{2}\right)$ were extracted using robust multi-array analysis $[53,54]$ and background correction was performed using perfect matches only which is implemented in the Bioconductor software package affy [55]. The linear model-fitting function in the affyPLM package [55] was used for quality assessment which was passed by all hybridized ATH1 GeneChips. One-way ANOVA (Bioconductor package manova [56]) was used to compute $p$-values of genes $(p \leq 0.05)$. TAIR9 (May 2009 update) gene functional descriptions were employed [57]. MapMan (version 3.1.1 [41]) was used to identify functional processes that were altered statistically $(P 0.05)$ in the developmental gene sets.

\section{Harvest of plant material, RNA extraction and CDNA synthesis for qPCR experiments}

The developmental stages of seeds were confirmed as described for Affymetrix GeneChip experiments. For plant harvest we concentrated on the main stages of embryogenesis and the silique length which are correlated in Table 1. Batches of up to $100 \mathrm{mg}$ of flowers and developing siliques from stages 0-10 (see Table 1), and rosette leaves, were harvested on dry ice. Additionally, siliques from developmental stages 6-10 were opened and separated into isolated seeds and open siliques consisting of valves and replum. Plant material was immediately frozen in liquid nitrogen and stored at $-80^{\circ} \mathrm{C}$ until RNA isolation. Total RNA extractions, including DNase treatment, were performed using the RNeasy Plant Mini Kit (Qiagen, Hilden, Germany) following the manufacturer's instructions. RNA concentration was quantified using a Nanodrop 1000 (Thermo Scientific, Wilmington, DE, USA). cDNA was synthesized from $2 \mu \mathrm{g}$ of total RNA as previously described by Mehrtens et al. [58].

\section{qPCR and data analyses}

Primers used for qPCR experiments were designed using ProbeFinder 2.45 (ROCHE Diagnostics-Applied Science, Mannheim, Germany) and are listed in additional file 2. qPCR was performed using the Platinum SYBR Green qPCR SuperMix UDG Kit (Invitrogen, Karlsruhe, Germany) following the manufacturer's instructions on a Rotor-Gene 6000 cycler (Qiagen, Hilden, Germany). For qPCR, cDNA from all biological samples were run in triplicate with cDNA from rosette leaves as the internal standard. Based on expression data from the AtGenExpress silique and seed developmental series [29] and Genevestigators' RefGenes tool (version V3 [59]), $A$. thaliana TRANSLOCASE INNER MEMBRANE SUBUNIT 44-2 (TIM44-2 [60]) was selected as the reference gene for qPCR normalization. TIM44-2 exhibited stable expression with minimal variation of the corrected crossing points (cCPs) across samples (Additional file 1) in pilot experiments and is therefore an excellent reference gene in accordance with MIQE guidelines [61]. Raw data was analyzed with the CAmpER software tool 
(Blom, personal communication [62]), which implements an algorithm based on a four parametric logistic model [63] of the fluorescence curve to calculate efficiency $\mathrm{CCPs}$ that were subsequently analyzed using the REST software [64]. Visualizations were created using Microsoft Office Excel 2007. Calculation of Pearson product-moment correlation coefficients was performed using Microsoft Office Excel 2007 according to Rodgers and Nicewander [65].

\section{Vanillin assay}

Vanillin staining of intact seeds was performed according to Debeaujon et al. [15] to color monomers and terminal subunits of PAs, such as leucoanthocyanidins and catechins [66].

\section{Additional material}

Additional file 1: Corrected crossing points of selected genes. QPCR efficiency corrected crossing points of the reference gene and three selected genes to verify organ specificity.

Additional file 2: Primers used in qPCR. Primer pairs (forward and reverse primer) for all genes used for qPCR [69].

\section{Acknowledgements}

CKK would like to thank the International NRW Graduate School in Bioinformatics and Genome Research for providing financial support. The authors wish to thank Ute Tartler, Melanie Kuhlmann, and Andrea Voigt for assistance with plant growth and harvest. We also thank Thomas Altmann, Lutz Nover, and Detlef Weigel for organizing the AtGenExpress consortium on which we rely on. We would like to acknowledge Oliver Jahns, Micheal Dondrup, and Jochen Blom for excellent technical support, as well as Helge Küster for critical reading and providing useful suggestions to improve the manuscript. A special thank to Divya Chandran for critical reading of the manuscript and language improvement.

\section{Author details}

'Bielefeld University, Department of Biology, Genome Research, 33594 Bielefeld, Germany. ${ }^{2}$ International NRW Graduate School in Bioinformatics and Genome Research, Bielefeld University, 33594 Bielefeld, Germany.

\section{Authors' contributions}

CKK analyzed the ATH1 GeneChip experiment data, performed the aPCR experiments and the corresponding work, such as plant harvest, RNA isolation, and wrote the manuscript. FM produced and characterized the plant material and the RNA samples for ATH1 GeneChip hybridizations. RS and BW provided the application cases, supervised, and directed the whole project. All authors read and approved the final manuscript.

\section{Competing interests}

The authors declare that they have no competing interests.

Received: 27 April 2010 Accepted: 7 October 2010

Published: 7 October 2010

\section{References}

1. Winkel-Shirley B: Flavonoid biosynthesis. A colorful model for genetics, biochemistry, cell biology, and biotechnology. Plant Physiology 2001, 126:485-493.

2. Clegg MT, Durbin ML: Flower color variation: a model for the experimental study of evolution. Proceedings of the National Academy of Sciences of the United States of America 2000, 97(13):7016-7023.
3. Lepiniec L, Debeaujon I, Routaboul JM, Baudry A, Pourcel L, Nesi N, Caboche M: Genetics and biochemistry of seed flavonoids. Annual Review of Plant Biology 2006, 57:405-430.

4. Li J, Ou-Lee T-M, Raba R, Amundson RG, Last RL: Arabidopsis flavonoid mutants are hypersensitive to UV-B irradiation. The Plant Cell 1993, 5:171-179.

5. Landry LG, Chapple CCS, Last RL: Arabidopsis mutants lacking phenolic sunscreens exhibit enhanced ultraviolet-B injury and oxidative damage. Plant Physiology 1995, 109(4):1159-1166.

6. Grotewold E: The genetics and biochemistry of floral pigments. Annual Review of Plant Biology 2006, 57:761-780.

7. Harborne JB: The Flavonoids: advances in research since 1980. New York: Chapman and Hall 1988.

8. Devic M, Guilleminot J, Debeaujon I, Bechtold N, Bensaude E, Koornneef M, Pelletier G, Delseny M: The BANYULS gene encodes a DFR-like protein and is a marker of early seed coat development. The Plant Journal 1999, 19:387-398.

9. Debeaujon I, Peeters AJM, Léon-Kloosterziel KM, Koornneef M: The TRANSPARENT TESTA12 gene of Arabidopsis encodes a multidrug secondary transporter-like protein required for flavonoid sequestration in vacuoles of the seed coat endothelium. The Plant Cell 2001, 13(4):853-872.

10. Drews GN, Yadegari R: Development and function of the angiosperm female gametophyte. Annual Review of Genetics 2002, 36:99-124.

11. Bowman J: Arabidopsis: An atlas of morphology and development. New York: Springer 1994.

12. Chaudhury AM, Craig S, Dennis E, Peacock W: Ovule and embryo development, apomixis and fertilization. Current Opinion in Plant Biology 1998, 1(1):26-31.

13. Hennig L, Gruissem W, Grossniklaus U, Köhler C: Transcriptional Programs of Early Reproductive Stages in Arabidopsis. Plant Physiology 2004, 135:1765-1775.

14. de Folter S, Busscher J, Colombo L, Losa A, Angenent GC: Transcript profiling of transcription factor genes during silique development in Arabidopsis. Plant Molecular Biology 2004, 56(3):351-366.

15. Debeaujon I, Leon-Kloosterziel K, Koornneef M: Influence of the testa on seed dormancy, germination, and longevity in Arabidopsis. Plant Physiology 2000, 122(2):403-413.

16. Debeaujon I, Nesi N, Perez P, Devic M, Grandjean O, Caboche M, Lepiniec L: Proanthocyanidin-accumulating cells in Arabidopsis testa: regulation of differentiation and role in seed development. The Plant Cell 2003, 15(11):2514-2531.

17. Bürger D: Die morphologischen Mutanten des Göttinger ArabidopsisSortiments, einschließlich der Mutanten mit abweichender Samenfarbe. Arabidopsis Information Services 1971, 8:36-42.

18. Koornneef M: Mutations affecting the testa colour in Arabidopsis. Arabidopsis Information Services 1990, 27:1-4.

19. Xie DY, Sharma SB, Paiva NL, Ferreira D, Dixon RA: Role of anthocyanidin reductase, encoded by BANYULS in plant flavonoid biosynthesis. Science 2003, 299(5605):396-399.

20. Zhao J, Pang Y, Dixon RA: The Mysteries of Proanthocyanidin Transport and Polymerization. Plant Physiology 2010, 153:437-443.

21. Quattrocchio F, Baudry A, Lepiniec L, Grotewold E: The regulation of flavonoid biosynthesis. In The science of flavonoids. Edited by: Grotewold E. Columbus: The Ohio State University; 2006:97-122.

22. Nesi N, Debeaujon I, Jond C, Pelletier G, Caboche M, Lepiniec L: The $\Pi$ T8 gene encodes a basic helix-loop-helix domain protein required for expression of DFR and BAN genes in Arabidopsis siliques. The Plant Cell 2000, 12(10):1863-1878.

23. Nesi N, Jond C, Debeaujon I, Caboche M, Lepiniec L: The Arabidopsis $\Pi T 2$ gene encodes an R2R3 MYB domain protein that acts as a key determinant for proanthocyanidin accumulation in developing seed. The Plant Cell 2001, 13(9):2099-2114.

24. Baudry A, Heim MA, Dubreucq B, Caboche M, Weisshaar B, Lepiniec L: TT2, TT8, and TG1 synergistically specify the expression of BANYULS and proanthocyanidin biosynthesis in Arabidopsis thaliana. The Plant Journal 2004, 39(3):366-380.

25. Sagasser M, Lu G-H, Hahlbrock K, Weisshaar B: A. thaliana TRANSPARENT TESTA 1 is involved in seed coat development and defines the WIP subfamily of plant zinc finger proteins. Genes \& Development 2002, 16(1):138-149. 
26. Gonzalez A, Zhao M, Leavitt JM, Lloyd AM: Regulation of the anthocyanin biosynthetic pathway by the TTG1/bHLH/Myb transcriptional complex in Arabidopsis seedlings. The Plant Journal 2008, 53(5):814-827.

27. Dixon RA, Paiva NL: Stress-induced phenylpropanoid metabolism. The Plant Cell 1995, 7:1085-1097.

28. Taylor LP, Grotewold E: Flavonoids as developmental regulators. Current Opinion in Plant Biology 2005, 8(3):317-323.

29. Schmid M, Davison TS, Henz SR, Pape UJ, Demar M, Vingron M, Scholkopf B, Weigel D, Lohmann JU: A gene expression map of Arabidopsis thaliana development. Nature Genetics 2005, 37(5):501-506.

30. Barker CS, Griffin C, Dolganov GM, Hanspers K, Yang JY, Erle DJ: Increased DNA microarray hybridization specificity using sscDNA targets. BMC Genomics 2005, 6(57).

31. Canales RD, Luo Y, Willey JC, Austermiller B, Barbacioru CC, Boysen C, Hunkapiller K, Jensen RV, Knight CR, Lee KY, et al: Evaluation of DNA microarray results with quantitative gene expression platforms. Nature Biotechnology 2006, 24(9):1115-1122.

32. Barnett MJ, Toman CJ, Fisher RF, Long SR: A dual-genome Symbiosis Chip for coordinate study of signal exchange and development in a prokaryote-host interaction. Proceedings of the National Academy of Sciences of the United States of America 2004, 101(47):16636-16641.

33. Tesfaye M, Silverstein KAT, Bucciarelli B, Samac DA, Vance CP: The Affymetrix Medicago GeneChip ${ }^{\circledR}$ array is applicable for transcript analysis of alfalfa (Medicago sativa). Functional Plant Biology 2006, 33(8):783-788.

34. Larkin JE, Frank BC, Gavras H, Sultana R, Quackenbush J: Independence and reproducibility across microarray platforms. Nature Methods 2005, 2(5):337-344.

35. Elbez $Y$, Farkash-Amar S, Simon I: An analysis of intra array repeats: the good, the bad and the non informative. BMC Genomics 2006, 5(7).

36. Eklund AC, Szallasi Z: Correction of technical bias in clinical microarray data improves concordance with known biological information. Genome Biology 2008, 9(2)

37. Skvortsov D, Abdueva D, Curtis C, Schaub B, Tavaré S: Explaining differences in saturation levels for Affymetrix GeneChip arrays. Nucleic Acids Research 2007, 35(12):4154-4163.

38. Nuccio ML, Thomas TL: ATS1 and ATS3: two novel embryo-specific genes in Arabidopsis thaliana. Plant Molecular Biology 1999, 39(6):1153-1163.

39. Rajani S, Sundaresan V: The Arabidopsis myc/bHLH gene ALCATRAZ enables cell separation in fruit dehiscence. Current Biology 2001, 11(24):1914-1922.

40. Takahashi S, Katagiri T, Yamaguchi-Shinozaki K, Shinozaki K: An Arabidopsis gene encoding a Ca2+-binding protein is induced by abscisic acid during dehydration. Plant Cell Physiology 2000, 41(7):898-903.

41. Thimm $O$, Bläsing $O$, Gibon $Y$, Nagel A, Meyer S, Krüger $P$, Selbig J, Müller LA, Rhee SY, Stitt M: MAPMAN: a user-driven tool to display genomics data sets onto diagrams of metabolic pathways and other biological processes. The Plant Journal 2004, 37(6):914-939.

42. Pourcel L, Routaboul JM, Kerhoas L, Caboche M, Lepiniec L, Debeaujon I: TRANSPARENT TESTA10 encodes a laccase-like enzyme involved in oxidative polymerization of flavonoids in Arabidopsis seed coat. The Plant Cell 2005, 17(11):2966-2980.

43. Albert $S$, Delseny $M$, Devic M: BANYULS, a novel negative regulator of flavonoid biosynthesis in the Arabidopsis seed coat. The Plant Journal 1997, 11(2):289-299.

44. Nesi N, Debeaujon I, Jond C, Stewart AJ, Jenkins Gl, Caboche M, Lepiniec L: The TRANSPARENT TESTA16 locus encodes the ARABIDOPSIS BSISTER MADS domain protein and is required for proper development and pigmentation of the seed coat. The Plant Cell 2002, 14(10):2463-2479.

45. Walker AR, Davison PA, Bolognesi-Winfield AC, James CM, Srinivasan N, Blundell TL, Esch JJ, Marks MD, Gray JC: The TRANSPARENT TESTA GLABRA1 locus, which regulates trichome differentiation and anthocyanin biosynthesis in Arabidopsis, encodes a WD40 repeat protein. The Plant Cell 1999, 11:1337-1350.

46. Tohge T, Nishiyama Y, Hirai MY, Yano M, Nakajima J, Awazuhara M, Inoue E, Takahashi H, Goodenowe DB, Kitayama M, et al: Functional genomics by integrated analysis of metabolome and transcriptome of Arabidopsis plants over-expressing an MYB transcription factor. The Plant Journal 2005, 42(2):218-235.

47. [http://www.plantontology.org/].
48. Ruuska SA, Ohlrogge JB: Protocol for small-scale RNA isolation and transcriptional profiling of developing Arabidopsis seeds. BioTechniques 2001, 31(4):756-758, 752, 754.

49. Affymetrix : GeneChip ${ }^{\circledR}$ Expression Analysis. Santa Clara, CA, USA Affymetrix, Inc 2001.

50. [http://arabidopsis.org/servlets/TairObject? type=expression_set\&id=1006710873]

51. Dondrup M, Albaum SP, Griebel T, Henckel K, Jünemann S, Kahlke T, Kleindt CK, Küster H, Linke B, Mertens D, et al: EMMA 2-a MAGE-compliant system for the collaborative analysis and integration of microarray data. BMC Bioinformatics 2009, 10(50).

52. Gentleman RC, Carey VJ, Bates DM, Bolstad B, Dettling M, Dudoit S, Ellis B, Gautier L, Ge Y, Gentry J, et al: Bioconductor: open software development for computational biology and bioinformatics. Genome Biology 2004, $5(10)$.

53. Irizarry RA, Bolstad BM, Collin F, Cope LM, Hobbs B, Speed TP: Summaries of Affymetrix GeneChip probe level data. Nucleic Acids Research 2003, 31(4).

54. Irizarry RA, Hobbs B, Collin F, Beazer-Barclay YD, Antonellis KJ, Scherf U, Speed TP: Exploration, normalization, and summaries of high density oligonucleotide array probe level data. Biostatistics 2003, 4(2):249-264.

55. Gautier L, Cope L, Bolstad BM, Irizarry RA: affy-analysis of Affymetrix GeneChip data at the probe level. Bioinformatics 2004, 20(3):307-315.

56. $\mathrm{Xu} \mathrm{J}$, Cui $X$ : Robustified MANOVA with applications in detecting differentially expressed genes from oligonucleotide arrays. Bioinformatics 2008, 24(8):1056-1062.

57. Rhee SY, Beavis W, Berardini TZ, Chen G, Dixon D, Doyle A, GarciaHernandez M, Huala E, Lander G, Montoya M, et al: The Arabidopsis Information Resource (TAIR): a model organism database providing a centralized, curated gateway to Arabidopsis biology, research materials and community. Nucleic Acids Research 2003, 31(1):224-228.

58. Mehrtens F, Kranz H, Bednarek P, Weisshaar B: The Arabidopsis transcription factor MYB12 is a flavonol-specific regulator of phenylpropanoid biosynthesis. Plant Physiology 2005, 138(2):1083-1096.

59. Hruz T, Laule O, Szabo G, Wessendorp F, Bleuler S, Oertle L, Widmayer P, Gruissem W, Zimmermann P: Genevestigator v3: a reference expression database for the meta-analysis of transcriptomes. Advances in Bioinformatics 2008.

60. Lister R, Chew O, Lee MN, Heazlewood JL, Clifton R, Parker KL, Millar AH, Whelan J: A transcriptomic and proteomic characterization of the Arabidopsis mitochondrial protein import apparatus and its response to mitochondrial dysfunction. Plant Physiology 2004, 134(2):777-789.

61. Bustin SA, Benes V, Garson JA, Hellemans J, Huggett J, Kubista M, Mueller R, Nolan T, Pfaffl MW, Shipley GL, et al: The MIQE guidelines: minimum information for publication of quantitative real-time PCR experiments. Clinical Chemistry 2009, 55(4):611-622.

62. [http://www.cebitec.uni-bielefeld.de/groups/brf/software/camper_info/].

63. Tichopad A, Dilger M, Schwarz G, Pfaffl MW: Standardized determination of real-time PCR efficiency from a single reaction set-up. Nucleic Acids Research 2003, 31(20).

64. Pfaffl MW, Horgan GW, Dempfle L: Relative expression software tool (REST) for group-wise comparison and statistical analysis of relative expression results in real-time PCR. Nucleic Acids Research 2002, 30(9).

65. Rodgers JL, Nicewander WA: Thirteen Ways to Look at the Correlation Coefficient. The American Statistician 1988, 42(1):59-66.

66. Deshpande SS, Cheryan M, Salunkhe DK: Tannin analysis of food products. Critical Reviews in Food Science and Nutrition 1986, 24(4):401-449.

67. Koes RE, Quattrocchio F, Mol JNM: The flavonoid biosynthetic pathway in plants: function and evolution. BioEssays 1994, 16(2):123-132.

68. Routaboul JM, Kerhoas L, Debeaujon I, Pourcel L, Caboche M, Einhorn J, Lepiniec L: Flavonoid diversity and biosynthesis in seed of Arabidopsis thaliana. Planta 2006, 224:96-107.

69. [https://www.roche-applied-science.com/index.jsp].

doi:10.1186/1756-0500-3-255

Cite this article as: Kleindt et al:: Expression analysis of flavonoid biosynthesis genes during Arabidopsis thaliana silique and seed development with a primary focus on the proanthocyanidin biosynthetic pathway. BMC Research Notes 2010 3:255. 